\title{
The implicit affiliation motive moderates cortisol responses to acute psychosocial stress in high school students
}

\author{
Mirko Wegner $^{a, *}$, Julia Schüler ${ }^{a}$, Henning Budde ${ }^{b, c, * *}$ \\ a University of Bern, Institute of Sport Science, Switzerland \\ ${ }^{b}$ Medical School Hamburg, Faculty of Human Sciences, Department of Pedagogy, Germany \\ c Reykjavik University, School of Science and Engineering, Department of Sport Science, Iceland
}

\section{KEYWORDS \\ Cortisol; \\ Affiliation motive; \\ Psychosocial stress; \\ Physical stress; \\ Adolescents; \\ School}

\begin{abstract}
Summary It has been previously shown that the implicit affiliation motive - the need to establish and maintain friendly relationships with others - leads to chronic health benefits. The underlying assumption for the present research was that the implicit affiliation motive also moderates the salivary cortisol response to acute psychological stress when some aspects of social evaluation and uncontrollability are involved. By contrast we did not expect similar effects in response to exercise as a physical stressor. Fifty-nine high school students aged $M=14.8$ years were randomly assigned to a psychosocial stress (publishing the results of an intelligence test performed), a physical stress (exercise intensity of $65-75 \%$ of $\mathrm{HR}_{\max }$ ), and a control condition (normal school lesson) each lasting $15 \mathrm{~min}$. Participants' affiliation motives were assessed using the Operant Motive Test and salivary cortisol samples were taken pre and post stressor. We found that the strength of the affiliation motive negatively predicted cortisol reactions to acute psychosocial but not to physical stress when compared to a control group. The results suggest that the affiliation motive buffers the effect of acute psychosocial stress on the HPA axis.
\end{abstract}

\footnotetext{
* Corresponding author at: University of Bern, Institute of Sport Science, Fabrikstrasse 8, 3012 Bern, Switzerland. Tel.: +41 316315659 ; fax: +41316314631.

** Corresponding author at: MSH Medical School Hamburg, Am Kaiserkai 1, 20457 Hamburg, Germany.

E-mail addresses: mirko.wegner@ispw.unibe.ch (M. Wegner), henning.budde@medicalschool-hamburg.de, henningb@ru.is (H. Budde).
} 


\section{Introduction}

Both, psychosocial and physical stress affects the activity of the hypothalamic-pituitary-adrenal (HPA) axis resulting in an increased release of cortisol. For chronically increased activity of the HPA axis several negative effects have been found including for example impairment of health (Dickerson and Kemeny, 2004). Researchers in the field are highly interested in individual difference variables that moderate the cortisol response to psychological and physical stress (Kudielka et al., 2009). Past research suggested age, gender, smoking, coffee, or alcohol consumption as moderators of the cortisol response to stress (Kudielka et al., 2009). However, less research focused on moderating personality variables.

One important moderating personality variable is the implicit affiliation motive (need for affiliation, nAff). The implicit affiliation motive is defined as the need to establish or maintain friendly, warm, and close interpersonal relationships (Murray, 1938; Atkinson et al., 1954; McAdams, 1980; McAdams and Constantian, 1983). Characteristics of the implicit affiliation motive are that it energizes behavior toward actively pursuing affiliative goals, it orients a person toward affiliative cues and it makes a person learn better (e.g., social networks) what is needed to reach an affiliation goal (Biernat, 1989; Koestner and McClelland, 1992). Baumeister and Leary (1995) suggest that the need to belong is a universal drive for frequent, affectively pleasant, stable and enduring relationships with others which includes concern for each other's welfare (p. 498). It is also suggested that this need for affiliation has an evolutionary base because of its benefits for reproduction and survival (e.g., care for offspring, food, hunting, protection) (Axelrod and Hamilton, 1981; Ainsworth, 1989). For this reason it is evolutionarily functional that the satisfaction of the affiliation motive has positive affective consequences and the dissatisfaction results in negative affect states (McClelland, 1985; McAdams and Bryant, 1987; Baumeister and Leary, 1995; Schüler et al., 2008). In addition to these emotional consequences, the implicit affiliation motive also possesses the qualities of a fundamental human need because it elicits goal-oriented behavior directed at satisfying the affiliation motive (Boyatzis, 1973) and has been linked to a variety of social behaviors (Lansing and Heyns, 1959; Exline, 1962; McAdams and Constantian, 1983).

The affiliation motive has been associated with chronic health benefits including, for example, decreased diastolic blood pressure (McClelland, 1979), increased immune function and decreased susceptibility to disease (Jemmott et al., 1990), fewer reported severity of illnesses (Mehta and Josephs, 2010) and a more positive perception of a neutral social interaction leading to increased well-being (Kordik et al., 2012). Other authors additionally focused on whether the implicit affiliation motive is also reflected in self-reports of personal affiliation values and goals (Brunstein et al., 1995; Schüler et al., 2008). They found that a congruence between self-reports of affiliation strivings and the implicit affiliation motive affected well-being positively. Moreover, different behavior associated with a dispositional need for affiliation (e.g., social interaction and support) has been previously shown to lead to protective health effects including decreased cortisol levels in research with primates
(Abbott et al., 2003) and humans alike (Thorsteinsson and James, 1999; Heinrichs et al., 2003). The latter research studies suggested that social affiliation and integration in a social group render situations that involve social evaluation less threatening and more controllable. Summing up, the dispositional desire to belong has several stress-reducing and therewith health-enhancing effects.

In previous studies it has not been tested whether the dispositional need for affiliation also results in a stress reducing effect without actually experiencing social interaction. Thus the aim of the present study was to test whether the implicit affiliation motive as an individual difference variable moderates the individual's cortisol response to acute psychosocial stress. To aim this we compared the cortisol response to psychosocial stress with the response to physical stress another typical stressor found in school - and to a control condition. To our knowledge, the stress-reducing effect of the implicit affiliation motive has not yet been reported in an adolescent sample.

\section{Materials and methods}

\subsection{Study design and data collection}

Participants filled in the implicit affiliation motive measure and completed questionnaires on sociodemographic variables before they participated in the test. Students were then randomly assigned to one of the three experimental groups. In the psychosocial stress group (PSY, $n=19$, female: 12) participants worked on verbal and mathematical tasks of an intelligence test (HAWIK-IV; Petermann and Petermann, 2006) for $15 \mathrm{~min}$. In order to induce psychosocial stress, students were told before they started working on the tasks that their IQ score would be made public at the end of the session. The tasks in the intelligence test exceeded all students' capability. The psychosocial stressor thus involved an aspect of social evaluation by peers and the experimenter as well as an achievement aspect because students assumed they could perform well or poorly. In the physical stress group (PHYS, $n=18$, female: 9) participants had to run in a noncompetitive mode at a moderate exercise intensity of $65-75 \%$ of their individual $\mathrm{HR}_{\max }$ for $15 \mathrm{~min}$. The control group (CON, $n=23$, female: 11$)$ took part in a teacher-centered lesson for the same amount of time. Salivary cortisol samples were taken before (pre) and after (post) being exposed to the stressor. Participants were told not to eat and drink $1 \mathrm{~h}$ before the testing took place.

\subsection{Study group}

Fifty-nine adolescents (32 female) aged $M=14.8$ years $(S D=0.5)$ from a high school in Berlin, Germany, participated in the study. Participants and their parents signed an informed consent form prior to participation. The present study was approved by the relevant ethics committee of the German Psychological Society (DGPs) and performed in accordance with the declaration of Helsinki. Exclusion criteria for the study were: dyslexia (verified by teachers' statements), obesity, physical or mental impairments, and the use of psychoactive substances (e.g. Ritalin). Findings of this study, but not related to the affiliation motive were 
previously reported (Wegner et al., 2014). None of the participants indicated that they were smokers or used oral contraceptives.

\subsection{Assessments}

\subsubsection{Cortisol}

Two saliva samples (pre, post) were taken using Salivettes with the blue cap (Sarstedt, Nümbrecht, Germany). Participants chewed on a synthetic swab for $1 \mathrm{~min}$. Afterwards the swabs were placed in the plastic tube of the Salivettes. Participants provided their first sample after the fourth school lesson at $1200 \mathrm{~h}$ and the second sample 30 min later. Samples were stored at $-20^{\circ} \mathrm{C}$ until they were analyzed. Biochemical analyses were performed at the CharitéUniversitätsmedizin, Berlin, Germany. A chemiluminescence immunoassay procedure (IBL, Hamburg, Germany) was used to assess cortisol levels in the samples taken. The intra- and inter-assay coefficients were below $8 \%$.

\subsubsection{Implicit affiliation motive}

To measure the implicit affiliation motive (nAff) the Operant Motive Test (OMT; Kuhl and Scheffer, 1999) was administered. The test consists of 15 pictures showing sketches of people e.g. talking to each other. Participants have to choose one of the people depicted as a protagonist first. Subsequently, they have to answer four questions spontaneously in note form. These four questions are: (1) What is important for the person in this situation and what is the person doing?, (2) How does the person feel?, (3) Why does the person feel this way? and (4) How does the story end? Participants' responses were coded using the standardized affiliation scoring rules of the OMT (Kuhl and Scheffer, 1999). According to the manual, the affiliation motive was coded whenever participants wrote about (A) friendly, intuitive and intimate exchange between friends, (B) having fun with others, (C) solving problems in affiliative or intimate relationships with others, (D) seeking security in the relationship with others or $(E)$ feelings of being alone. Whole pictures were either coded or not coded for the affiliation motive. Two experienced coders reached satisfactory interand intra-rater reliabilities of .85 and .88 , respectively. Affiliation scores ranged from 0 to $7(M=3.48, S D=1.31)$. For subsequent data analyses the affiliation scores were $z$ standardized. The implicit affiliation motive assessed using the OMT has been previously linked to right hemispheric activity (Kuhl and Kazén, 2008) and friendly behavior toward customers (Scheffer et al., 2007).

\subsection{Statistical analysis}

A series of hierarchical regression analyses were conducted to control for lower order effects before testing for higher order effects. The motive measure (OMT) was used as the quantitative continuous independent variable measuring individual differences in participants' implicit affiliation motive. Experimental group allocations were used as categorical independent variables and combined to interaction terms with the affiliation motive. The post-stressor salivary cortisol level served as the dependent variable. Pre-stressor cortisol and gender were entered as covariates (see Table 2).
Table 1 Cortisol raw scores $(\mathrm{ng} / \mathrm{ml})$ of the pre- and postmeasures for the physical stress, the psychosocial stress, and the control group.

\begin{tabular}{llllll}
\hline & \multicolumn{2}{l}{ Cortisol } & & \\
\cline { 2 - 3 } \cline { 5 - 6 } & \multicolumn{2}{l}{ Pre } & & \multicolumn{2}{l}{ Post } \\
\cline { 2 - 3 } \cline { 5 - 6 } & $M$ & $S D$ & & $M$ & $S D$ \\
\hline Physical stress & 1.74 & 0.66 & & 2.15 & 1.57 \\
Psychosocial stress & 1.89 & 0.86 & & 2.24 & 1.10 \\
Control & 1.45 & 0.78 & & 1.80 & 0.72 \\
\hline
\end{tabular}

All cortisol measures were log-transformed due to skewed distribution. The statistical analysis procedure used here has been previously applied (e.g., Schultheiss et al., 2014).

\section{Results}

\subsection{Descriptive statistics}

In preliminary analyses we found that the three experimental groups did not differ regarding age, $F(2,56)=0.71$, $p=.50$, or gender, $\chi^{2}(2)=1.10, p=.58$. Descriptive data of the pre- and post-stressor cortisol raw scores can be found in Table 1. According to Van Cauter and Refetoff (1985) a cortisol response is present when the cortisol pre-value is exceeded by more than $10 \%$. In our adolescent sample the average cortisol response from pre to post-test is approximately $20 \%, F(1,58)=3.80, p=.05, \eta_{p}^{2}=.06$.

\subsection{Regression analyses on the moderating effect of the affiliation motive on cortisol changes}

In order to test the moderating effect of the implicit affiliation motive on cortisol responses in the three experimental groups, we conducted four hierarchical regression models (see Table 2). Post-stressor cortisol scores were residualized for gender and pre-stressor cortisol levels in the first block (Model 1). This model revealed low regression weights between pre- and post-stressor levels of cortisol $(B=-0.18)$ and no gender effects on the cortisol response $(B=-0.07)$, as illustrated in Table 2. Consequently, the remaining three models now tested the effects of the affiliation motive (Model 2), the experimental groups (Model 3), and the interaction of implicit affiliation motive $\times$ experimental groups (Model 4) on the cortisol response residualized for pre-stressor cortisol scores. Thus, in Model 2 we tested the main effect of the implicit affiliation motive on the cortisol response. Although the whole model was not significant including the implicit affiliation motive improved the regression model significantly, $\Delta R^{2}=.069, \Delta F(1,55)=4.22$, $p=.045$, meaning adolescents with a high implicit affiliation motive showed lower cortisol levels after $30 \mathrm{~min}$ independent of the kind of stressor, $B=-0.098, S E=0.047, t=-2.05$, $p=.045$. In Model 3 we added the experimental conditions into the regression analysis, resulting in no improvement of the model. Finally (Model 4), including the interaction terms (multiplicative term of $z$-transformed variables) of the implicit affiliation motive $(n A f f) \times$ experimental 


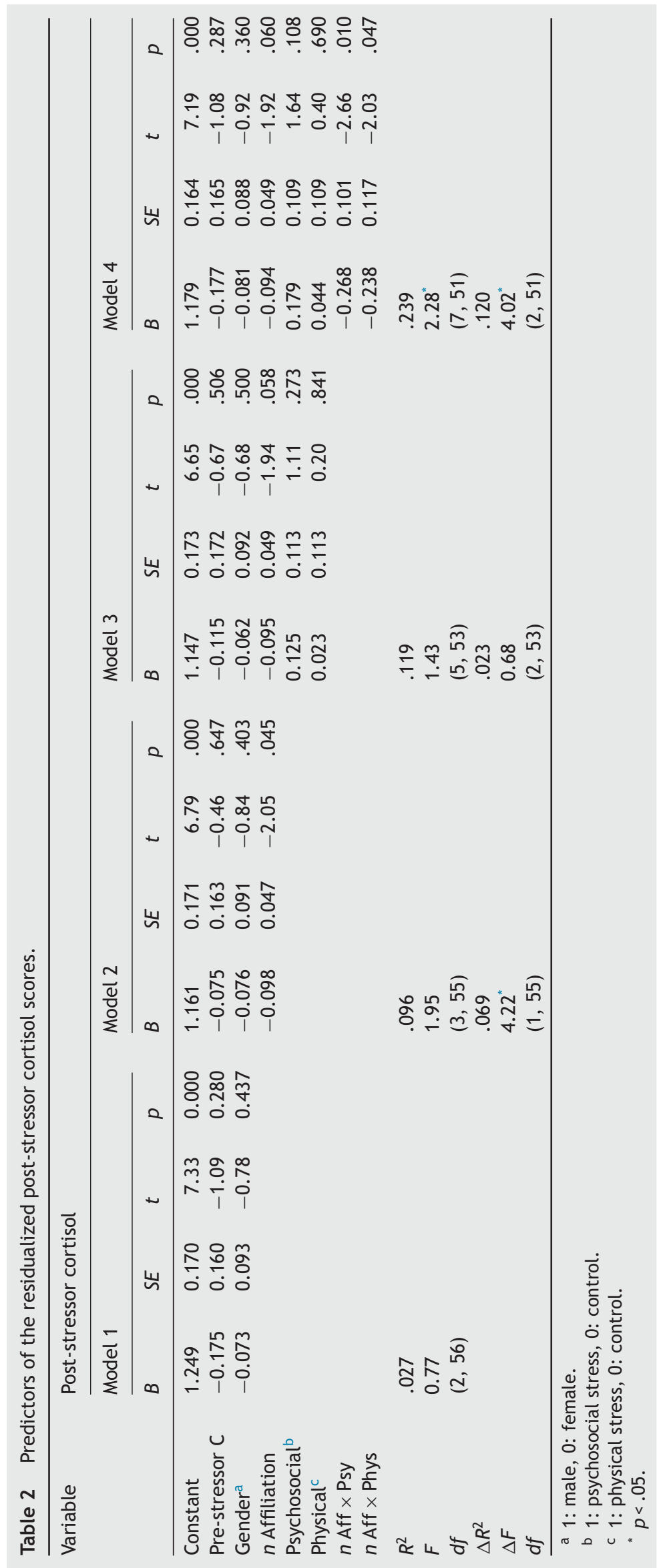




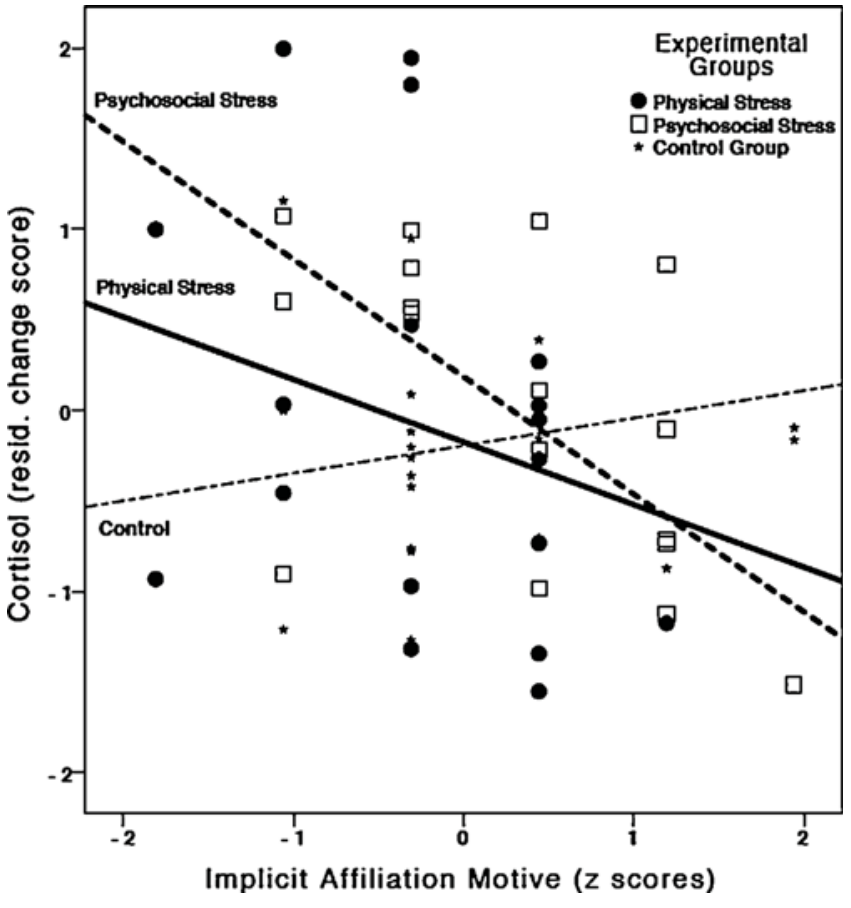

Figure 1 Association between the implicit affiliation motive and salivary cortisol responses (log-transformed) in the three experimental conditions (psychosocial stress - dashed line, physical stress - solid line, control - thin dashed line).

conditions rendered the whole model significant, $R^{2}=.239$, $F(7,51)=2.28, p=.042$ (see Table 2). This means that students' cortisol responses to the experimental conditions were dependent on their levels of implicit affiliation motive.

Follow-up regression analyses revealed that cortisol responses of participants in the psychosocial stress group were significantly negatively associated with the implicit affiliation motive, $B=-0.531$, $S E=0.156, p r=-.65$, $t(17)=-3.40, p=.004$ (see Fig. 1). This means that participants high in the affiliation motive showed lower cortisol levels in response to the psychosocial stressor used. In the physical stress condition, $B=-0.463, S E=0.156, p r=-.35$, $t(17)=-1.47, p=.161$, and the control condition cortisol responses were not significantly related to the affiliation motive, $B=0.096, S E=0.136, p r=.15, t(22)=0.71, p=.488$. All interaction effects are illustrated in Fig. 1.

\section{Discussion}

According to our hypothesis in the present study adolescents' cortisol responses to psychosocial stress in a school setting depended on their implicit affiliation motive. However, the affiliation motive did not moderate the effects of physical stress. Due to these results it seems that the affiliation motive is not important until the stress situation involves some aspect of social evaluation. Although neither the psychosocial nor the physical stressor alone were able to significantly predict cortisol responses (Model 3, Table 2 ) the regression coefficient of the psychosocial stressor $(B=0.125)$ was higher than the coefficient for physical stress $(B=0.023)$ suggesting a stronger effect on cortisol responses.
One reason why the affiliation motive may be able to moderate the psychosocial but not the physical stress response is given by Abbott et al. (2003). In their study with primates they argue that individuals differ in their psychological stress response if they lack information about timing, severity, or duration of the psychological stressor. In this work the psychological stress response is attributed to rank-related stress. A primate that has to fear rejection within a group will experience less psychological stress if it is well rooted in the group's social network, which also functions as a coping outlet for experienced stress. Psychological stress resulting from in-group rejection will then be more predictable for an individual who knows how to establish stable and predictable social relations; or is high in its "affiliation motive" (cf. Abbott et al., 2003). Similar results could be previously found in humans (Heinrichs et al., 2003). In this study, social support led to a decreased cortisol reaction to acute psychological stress. Heinrichs and colleagues also attributed their findings to participants' appraisal of the acute stress situation as less threatening and more controllable. Different to our present study they looked at actual social support but not at the affiliation motive as an individual difference variable. For mere physical stressors an affiliative competence does not help to obtain information about the controllability of the stressor.

Still little is known about how social support actually reduces cortisol on a neurophysiological level. From animal and human research it is known that beyond its central role as social attachment regulator oxytocin (Heinrichs et al., 2003) but also progesterone (Wirth and Schultheiss, 2006) inhibit the activity of the HPA axis responsiveness. Future studies may benefit from including oxytocin or progesterone measures in the study design. However, the administration of oxytocin remains problematic because of ethical questions about potentially persisting behavioral effects since there are reports of oxytocin levels remaining increased for at least $7 \mathrm{~h}$ (van IJzendoorn et al., 2012).

In our present analysis we also found that merely including the implicit affiliation motive (Model 2) significantly improved the regression model (Table 2 ), additionally pointing to the relevance of the affiliation motive independent of the type of stressor. This is in line with previous research findings relating cortisol levels to psychometric and behavioral measures of affiliation, but primarily in women. Wirth and Schultheiss (2006) for example related women's implicit affiliation motive to cortisol levels. However, in the present study the affiliation motive was related to adolescents' cortisol levels independent of gender.

Our results suggest that a high implicit affiliation motive is associated with reduced acute stress responses as expressed in students' cortisol levels. This is important especially in the light of previous findings on the chronic health benefits of the affiliation motive (e.g., Jemmott et al., 1990; Mehta and Josephs, 2010). However, it could also be argued that the reduced cortisol response to the intelligence testing in our data represents a maladaptive response to an achievement situation (Erickson et al., 2003). For example it has been previously shown that cortisol elevation could benefit performances in areas like perception (Seckl et al., 1991; McGaugh, 2000; Buchanan and Lovallo, 2001), rewardrelated learning, or psychomotor activity (Piazza and Le Loal, 1996). 
The present study focused on an adolescent sample in order to increase the knowledge base on stress responses in this age group. It is well known that age, pubertal stage, and emotional functioning can affect cortisol activity in this age group and that the HPA axis is subject to developmental change (Adam, 2006). Thus, results from our study may be generalized only with caution to similar age groups or adult samples.

In our study typical modifiers like age or gender had no effect on the results. Gender was also not found to significantly affect cortisol levels in other studies examining similar samples (Adam, 2006; Budde et al., 2010a,b). Moreover, although one might expect that women would score higher in their implicit affiliation motive, research regarding this topic did not draw a consistent picture with some authors (Exline, 1960) reporting gender differences regarding the implicit affiliation motive and others did not (McAdams and Constantian, 1983; Koestner and McClelland, 1992). In our sample girls' implicit affiliation motives were not higher than boys' affiliation motives.

\subsection{Limitations and future directions}

The present study is subject to some limitations. One limitation is that only two saliva cortisol samples were taken. The samples were taken before and after the presentation of the psychosocial and the physical stressor. In other studies up to eight cortisol samples were assessed to being able to analyze the cortisol reaction pattern (e.g., Heinrichs et al., 2003). However, from previous studies on the kinetics of cortisol we concluded that the post-test performed 30 min past the onset of the stressor would be an adequate time interval to approximately measure cortisol peaks. Additionally, overall cortisol levels of this adolescent sample are rather low. Nevertheless, these values are comparable with other research studies examining adolescents' cortisol levels in a similar age group (Budde et al., 2010a,b).

Moreover, within the present study we did not ask participants for their perceived social support or how satisfied they were with their everyday social relationships. Future studies should include implicit affiliation motive measures and measures of perceived social support especially when interested in chronic differences between individuals high and low in the implicit affiliation motive. Moreover, future research should also include a measure of the psychological stress reaction for example as reported in a self-report of affect or self-perceived stress.

Taken together, our results suggest that the implicit affiliation motive is an important moderating variable for the cortisol response to psychosocial stress. Both stressors used in the present study, psychosocial and physical, are relevant stressors found in a school setting.

Combining our results with previous findings (e.g., Heinrichs et al., 2003) suggests that social support and the implicit affiliation motive - as the need for social interaction - may contribute to buffering students' stress responses in school.

\section{Contributors}

MW and HB designed the study and wrote the protocol. MW managed the literature searches and analyses. MW and JS undertook the statistical analysis, and author $\mathrm{HB}$ and $\mathrm{MW}$ wrote the first draft of the manuscript. All authors contributed to and have approved the final manuscript.

\section{Role of the funding source}

This work was supported by funds from the Reykjavik University, Iceland, which had no further role in the study design; collection, analysis, and interpretation of data; in writing of the report; or in the decision to submit the paper for publication.

\section{Conflicts of interest}

The authors have no conflicts of interest and declare no financial interests. All authors disclose any actual or potential conflict of interest including any financial, personal or other relationships with other people or organizations within three years of beginning the work submitted that could inappropriately influence, or be perceived to influence, their work.

\section{Acknowledgements}

Henning Budde is supported by the German Research Foundation (DFG, Deutsche Forschungsgemeinschaft); BU 1837/5-1. We would like to thank Annemarie Mac Dowall (Department of Psychology, Medical School Hamburg) for proofreading.

\section{References}

Abbott, D.H., Keverne, E.B., Bercovitch, F.B., Shively, C.A., Mendoza, S.P., Saltzman, W., Snowdon, C.T., Ziegler, T.E., Banjevic, M., Garland, T.J., Sapolsky, R.M., 2003. Are subordinates always stressed? A comparative analysis of rank differences in cortisol levels among primates. Horm. Behav. 43, 67-82.

Adam, E.K., 2006. Transactions among adolescent trait and state emotion and diurnal and momentary cortisol activity in naturalistic settings. Psychoneuroendocrinology 31, 664-679.

Ainsworth, M.D., 1989. Attachments beyond infancy. Am. Psychol. 44, 709-716.

Atkinson, J.W., Heyns, R.W., Veroff, J., 1954. The effect of experimental arousal of the affiliation motive on thematic apperception. J. Abnorm. Psych. 49, 405-410.

Axelrod, R., Hamilton, W.D., 1981. The evolution of cooperation. Science 211, 1390-1396.

Baumeister, R.F., Leary, M.R., 1995. The need to belong: desire for interpersonal attachments as a fundamental human motivation. Psychol. Bull. 117, 497-529.

Biernat, M., 1989. Motives and values to achieve: different constructs with different effects. J. Pers. 57, 69-95.

Boyatzis, R.E., 1973. Affiliation motivation. In: McClelland, D.C., Steele, R.S. (Eds.), Human Motivation: A book of Readings. General Learning Corporation, Morristown, pp. 252-276.

Brunstein, J.C., Lautenschlager, U., Nawroth, B., Pöhlmann, K., Schultheiss, O.C., 1995. Persönliche Anliegen, soziale Motive und emotionales Wohlbefinden (Personal goals, social motives, and emotional well-being). Z. Differ. Diagn. Psychol. 16, 1-10.

Buchanan, T.W., Lovallo, W.R., 2001. Enhanced memory for emotional material following stress-level cortisol treatment in humans. Psychoneuroendocrinology 26, 307-317. 
Budde, H., Pietrassyk-Kendziorra, S., Bohm, S., Voelcker-Rehage, C., 2010a. Hormonal responses to physical and cognitive stress in a school setting. Neurosci. Lett. 474, 131-134.

Budde, H., Voelcker-Rehage, C., Pietrassyk-Kendziorra, S., Machado, S., Ribeiro, P., Arafat, A.M., 2010b. Steroid hormones in the saliva of adolescents after different exercise intensities and their influence on working memory in a school setting. Psychoneuroendocrinology 35, 382-391.

Dickerson, S.S., Kemeny, M.E., 2004. Acute stressors and cortisol responses: a theoretical integration and synthesis of laboratory research. Psychol. Bull. 130, 355-391.

Erickson, K., Drevets, W., Schulkin, J., 2003. Glucocorticoid regulation of diverse cognitive functions in normal and pathological emotional states. Neurosci. Biobehav. Rev. 27, 233-246.

Exline, R.V., 1960. Effects of sex, norms, and affiliation motivation upon accuracy of perception of interpersonal preferences. J. Pers. 28, 397-412.

Exline, R.V., 1962. Need affiliation and initial communication behavior in problem-solving groups characterized by low interpersonal visibility. Psychol. Rep. 10, 79-89.

Heinrichs, M., Baumgartner, T., Kirschbaum, C., Ehlert, U., 2003. Social support and oxytocin interact to suppress cortisol and subjective responses to psychosocial stress. Biol. Psychiatry 54, 1389-1398.

Jemmott, J.B., Hellman, C., McClelland, D.C., Locke, S.E., Kraus, L., Williams, R.M., Valeri, C.R., 1990. Motivational syndromes associated with natural killer cell activity. J. Behav. Med. 13, 53-73.

Koestner, R., McClelland, D.C., 1992. The affiliation motive. In: Smith, C.P. (Ed.), Motivation and Personality: Handbook of Thematic Content Analysis. Cambridge University Press, New York, pp. 205-210.

Kordik, A., Eska, K., Schultheiss, O.C., 2012. Implicit need for affiliation is associated with increased corrugator activity in a non-positive, but not in a positive social interaction. J. Res. Pers..

Kudielka, B.M., Hellhammer, D.H., Wüst, S., 2009. Why do we respond so differently? Reviewing determinants of human salivary cortisol responses to challenge. Psychoneuroendocrinology 34, 2-18.

Kuhl, J., Kazén, M., 2008. Motivation, affect, and hemispheric asymmetry: power versus affiliation. J. Pers. Soc. Psychol. 95, 456-469.

Kuhl, J., Scheffer, D., 1999. Der Operante Multi-Motiv-Test (OMT): Manual (The Operant Multimotive Test (OMT): Manual). University of Osnabrück, Osnabrück, Germany.

Lansing, J.B., Heyns, R.W., 1959. Need affiliation and frequency of four types of communication. J. Abnorm. Soc. Psych. 58, $365-372$.

McAdams, D.P., 1980. A thematic coding system for the intimacy motive. J. Res. Pers. 14, 413-432.

McAdams, D.P., Bryant, F.B., 1987. Intimacy motivation and subjective mental health in a nationwide sample. J. Pers. 55, 395-413.
McAdams, D.P., Constantian, C.A., 1983. Intimacy and affiliation motives in daily living: an experience sampling analysis. J. Pers. Soc. Psychol. 45, 851-861.

McClelland, D.C., 1979. Inhibited power motivation and high blood pressure in men. J. Abnorm. Psychol. 88, 182-190.

McClelland, D.C., 1985. Human Motivation. Foresman \& Co., Glenview, Scott.

McGaugh, J.L., 2000. Memory - a century of consolidation. Science 287, 248-251.

Mehta, P.H., Josephs, R.A., 2010. Social endocrinology: hormones and social motivation. In: Dunning, D. (Ed.), Social Motivation. Taylor and Francis, New York, pp. 171-190.

Murray, H.A., 1938. Explorations in Personality. Oxford University Press, New York.

Petermann, F., Petermann, U., 2006. Hamburg-WechslerIntelligenztest für Kinder (HAWIK-IV) (Hamburg-WechselIntelligence Test for children (HAWIK-IV)). Huber, Bern.

Piazza, P.V., Le Loal, M.L., 1996. Pathophysiological basis of vulnerability to drug abuse: role of an interaction between stress, glucocorticoids, and dopaminergic neurons. Annu. Rev. Pharmacol. Toxicol. 36, 359-378.

Scheffer, D., Eichstaedt, J., Chasiotis, A., Kuhl, J., 2007. Towards an integrated measure of need affiliation and agreeableness derived from the Operant Motive Test. Psychol. Sci. 49, 308-324.

Schüler, J., Job, V., Fröhlich, S.M., Brandstätter, V., 2008. A high implicit affiliation motive does not always make you happy: a corresponding explicit motive and corresponding behavior are further needed. Motiv. Emot. 32, 231-242.

Schultheiss, O.C., Wiemers, U.S., Wolf, O.T., 2014. Implicit need for achievement predicts attenuated cortisol responses to difficult tasks. J. Res. Pers. 48, 84-92.

Seckl, J.R., Dickson, K.L., Yates, C., Fink, G., 1991. Distribution of glucocorticoid and mineralcorticoid receptor messenger RNA expression in human postmortem hippocampus. Brain Res. 561, 332-337.

Thorsteinsson, E.B., James, J.E., 1999. A meta-analysis of the effects of experimental manipulations of social support during laboratory stress. Psychol. Health 14, 869-886.

Van Cauter, E., Refetoff, S., 1985. Evidence of two subtypes of cushing's disease based on the analysis of episodic cortisol secretion. New Engl. J. Med. 312, 1343-1349.

van IJzendoorn, M.H., Bhandari, R., van der Veen, R., Grewen, K.M., Bakermans-Kranenburg, M.J., 2012. Elevated salivary levels of oxytocin persist more than $7 \mathrm{~h}$ after intranasal administration. Front. Neuroendocrinol. 6, 1-6.

Wegner, M., Koedijker, J.M., Budde, H., 2014. The effect of acute exercise and psychosocial stress on fine motor skills and testosterone concentration in the saliva of high school students. PLoS ONE 9, e92953.

Wirth, M.M., Schultheiss, O.C., 2006. Effects of affiliation arousal (hope of closeness) and affiliation stress (fear of rejection) on progesterone and cortisol. Horm. Behav. 50, 786-795. 